\title{
Presumed Herpes simplex virus reactivation following LASIK mimicking diffuse lamellar keratitis
}

Arjun Srirampur', Prashant Garg ${ }^{2}$, Gangaprasad Amula ${ }^{2}$

'L V Prasad Eye Institute, Kode Venkatadri Chowdary Campus, Tadigadapa, Vijayawada Andhra Pradesh, India; ${ }^{2}$ V V Prasad Eye Institute, Kallam Anji Reddy Campus, Banjara Hills, Hyderabad, India

\begin{abstract}
We report a rare presentation of herpetic stromal keratitis reactivation in a 43-yearold patient who underwent LASIK previously and presented with complaints of photophobia, pain, and redness. On examination, mild congestion was noted with deep-seated, ring-like infiltrate under the LASIK flap with some amount of scarring that was initially confused with diffuse lamellar keratitis. A detailed repeated history led to diagnosing herpetic keratitis reactivation that with timely treatment of antivirals and steroids helped us to achieve a good anatomic and visual outcome for the patient.
\end{abstract}

Keywords: diffuse lamellar keratitis (DLK), herpetic stromal keratitis, LASIK

\section{Introduction}

The success of LASIK is attributed to its effectiveness, rapid visual recovery, and relatively uncommon complications like epithelial ingrowth, flap irregularities, regression, and diffuse lamellar keratitis (DLK). Rare, severe complications include infectious keratitis and corneal perforation. ${ }^{1}$

Herpes simplex (HSV) keratitis following LASIK and photorefractive keratectomy (PRK) has been demonstrated in animal experiments but few cases have been reported in humans. ${ }^{2}$ We present here a case of reactivation of HSV keratitis following excimer laser treatment.

\section{Case history}

A 43-year-old female presented with complaints of pain, redness, and photophobia in the left eye for the last three months. Her medical history indicated an immunocompetent woman without any comorbid conditions.

Ocular history revealed she had undergone LASIK in both eyes at a private clinic five months back, the details of which were not available. Her symptoms started

Correspondence: Dr. Arjun Srirampur, L V Prasad Eye Institute, Kode Venkatadri

Chowdary Campus, Tadigadapa, Vijayawada Andhra Pradesh, India.

E-mail: sarjuneye@gmail.com 


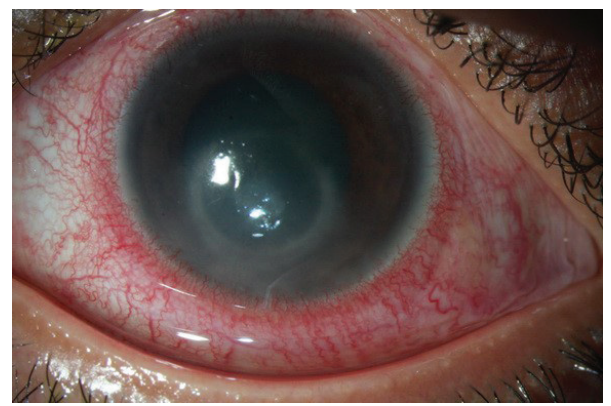

Fig. 1. Slit lamp photograph of the left eye at the time of presentation.

two months post LASIK in the left eye; it was diagnosed as DLK and treated with topical steroids. She had recurrent attacks of red eye with discontinuation of steroids.

At presentation, her best spectacle corrected visual acuity (BSCVA) was 20/20 in the right eye and 20/25 in the left eye. Intraocular pressure was normal. Right eye had a LASIK flap well apposed and the left eye had mild congestion, deep-seated ring-like infiltrate under the LASIK flap with some amount of scarring (Fig. 1). Posterior segment in both eyes was normal.

Clinical impression was DLK in the left eye, but as it was persisting for more than three months, infection had to be ruled out, especially atypical mycobacterium and other slow-growing organisms. Next day left eye LASIK flap lift with corneal scraping was done for Gram stain, $\mathrm{KOH}$ - Calcoflor white, 1\% \& 20\% Zeil-Neilson stain, blood agar, chocolate agar, potato dextrose agar, Sabouraud dextrose agar, Loweinstein-Jensen media, non-nutrient agar, and brain heart infusion agar under local anaesthesia in aseptic conditions. Smears revealed no organisms; hence, the patient was put on chloramphenicol eye drops 4 times/day and atropine eye drops 3 times/day. Patient was reviewed next day with a visual acuity of 20/100. As there was no growth in the culture media, she was continued on similar treatment. She was seen again after three days and her visual acuity had decreased to 20/160 with increase in the cellularity under the flap.

As she had some amount of scarring with deep vessels, the history was reviewed again, revealing that she had an attack of red eye in the same eye 10-12 years back, with a residual small scar. Reactivation of HSV keratitis after LASIK was suspected and she was put on oral acyclovir $400 \mathrm{mg}, 5$ times/day along with prednisolone eye drops 4 times /day and atropine eye drops 3 times/day.

She was seen after four days with improvement of symptoms and visual acuity of 20 /40 with decreased cellular infiltrates. Subsequently, she was continued on similar treatment for two weeks. After two weeks she had a visual acuity of 20/30, 


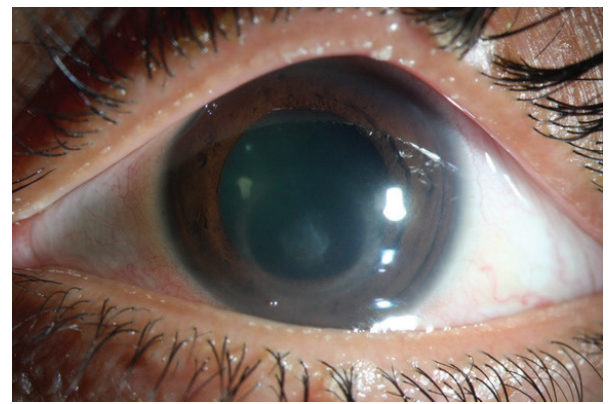

Fig. 2. Slit lamp photograph of the left eye after two months.

improving to $20 / 20$ with decreased cellularity. She was advised to continue tab acyclovir $400 \mathrm{mg} 2$ times/day, tapering prednisolone eye drops and asked to follow-up after two months.

After two months (Fig. 2) she had no infiltrate, scarred interface with visual acuity of $20 / 20$, and she was asked to continue tab acyclovir 400 mg 2 times /day and prednisolone eye drops once daily. No reactivation of HSV keratitis was seen in this period.

\section{Discussion}

DLK is a LASIK-specific complication with multiple reports of late onset presentation that may occur several months to years after surgery.,4 Probst et al. has described late onset DLKseven months after an uneventful LASIK in a 30-year-old woman.4 Multiple factors leading to the development of DLK have been listed but the exact cause still remains unclear.

In our case, the primary surgeon treated along the lines of DLK management and, when after three months of treatment the issue remained unsettled, it was referred to our clinic, where a detailed history and examination lead us to a suspicion of HSV reactivation. The mechanism by which excimer laser may trigger reactivation of HSV type 1 is not known. ${ }^{5}$ Probably, damage or irritation of corneal nerves and exposure to ultraviolet light with postoperative corneal inflammation may trigger a reactivation. ${ }^{6}$ It may also be that the use of corticosteroid in patients with history of HSV type 1 infection predisposes to reactivation.

\section{Conclusion}

This case represents an unusual form of HSV reactivation following LASIK, which was misdiagnosed and treated as late onset DLK, which to our best of knowledge has not been reported previously.

The case also punctuates the importance of noting detailed medical history 
regarding any previous red eye or corneal scars before embarking on laser refractive surgery. In case of any positive history or suspected HSV scar, patients should be on prophylactic acyclovir prophylaxis to prevent reactivation. This case also shows that routine therapeutic dose of acyclovir in the initial period followed by prophylactic dose later can be used in the management of HSV reactivation.

\section{References}

1. Perry HD, Doshi SJ. Herpes simplex reactivation following laser in situ keratomileusis and subsequent corneal perforation. CLAO J. 2002;28:69-71.

2. Dhaliwal DK, Romanowski. Experimental laser-assisted in situ keratomileusis induces the reactivation of latent herpes simplex virus. Am J Ophthalmol. 2001;131:506-507.

3. Jin GJ, Lyle WA, Merkley KH. Late-onset diffuse lamellar keratitis after a laser in situ keratomileusis. J Cataract Refract Surg. 2005;31(2):435-437.

4. Probst LE, Foley L. Late-onset interface keratitis after uneventful laser in situ keratomileusis. J Cataract Refract Surg. 2001;27(7):1124-1125.

5. Levy J, Lapid-Gortzak R. Herpes simplex virus keratitis after laser in situ keratomileusis. J Refract Surg. 2005; 21:400-402.

6. Davidorf JM. Herpes simplex keratitis after LASIK. J Refract Surg. 1998;4:667. 